\title{
Visualising Spatial Complexity of Urban Sites
}

\author{
Alan Mee \\ UCD School of Architecture Planning and Environmental Policy, \\ University College Dublin, \\ Richview School of Architecture, UCD Richview, Dublin 14, Ireland \\ alan@mee.ie
}

\begin{abstract}
Recent research has linked urban design to artistic practice, and it is suggested that urban design could become refocused if conceived of as an integrative art of place. However, the urban design research methods literature is silent on the topic of artistic practice, and therefore the potential for interpretative, abductive knowledge to emerge from creative visualisation in urban design is under-appreciated. More specifically, regarding evaluation for urban design, complexity theories of cities claim that understanding complexity in particular enhances our ability to organise knowledge in relation to cities, and it is further argued that complexity theory responds to a need for a knowledge leap in planning, design and maintenance of cities, to rise to global urban challenges and crises. In this context, visualising spatial complexity of urban sites through artistic practice is useful to urban design. The specifically spatial complexity of urban sites, (as the spatial component of urban complexity), has not previously been explored, evaluated or visualised for urban design. This paper argues that in creatively visualising spatial complexity, multi-scalar, relational and temporal aspects of the urban built environment can be captured, and this visualisation communicates a unique 'signature' of each urban site.
\end{abstract}

Keywords: Spatial complexity / urban design / artistic practice / visualisation.

\section{Introduction}

In this paper, a conceptual framework of visualising spatial complexity of urban sites is developed, to represent a prior evaluation which measures three issues and nine criteria of spatial complexity, in order to reveal and understand the relationships between compositional, configurational and systems aspects of urban sites. In the first section, related concepts of spatial complexity, representation and visualisation theory are briefly defined and discussed. The second section looks at the role of artistic practice in urban design visualisation. The third section sets out the visualisation approach of this study and the data transformation tasks undertaken. 
Then three key tools for visualisation of the evaluation of spatial complexity are proposed: Tables, Plots and Boxes. These devices are employed in order to capture the explored and evaluated conditions in a visually coherent complexity frame. Examples of these are illustrated, and the tools are compared in relation to the data dimensions spectrum of digital visualisation. Four particular benefits to urban design practice of employing appropriate visualisation of spatial complexity are suggested. Firstly, to stimulate urban design process: secondly, to bring visual clarity to quantitative assessment: thirdly, to allow qualitative interpretation by a wide community around evaluation, and lastly, to allow formal measurement and informal interpretation of results Following discussion of the visualisations presented, some conclusions and further recommendations are proposed in relation to visualising spatial complexity of urban sites.

\section{Spatial complexity, representation and visualisation theory}

The urban environment is complex [1], [2] and complexity is associated with data rich environments [3] like cities. In this context, information visualisation is transforming understandings of urban environments. Regarding evaluation for urban design, complexity theories of cities claim that understanding complexity in particular enhances our ability to organise knowledge in relation to cities, and it is further argued that complexity theory responds to a need for a knowledge leap in planning, design and maintenance of cities, to rise to global urban challenges and crises. In the spatial sciences, the concept of spatial complexity (the spatial component of urban complexity) has a large visual dimension, and has been categorised by pattern recognition in pixels of aerial views at large scales [4], classification of spatial complexity of rural landscapes [5] and recognition of fractal or geometrical patterns across population, remote sensed imagery and street network representations [6]. Research has sometimes implied that the visual dimension is the predominant property of spatial complexity [7] and the concept of visual complexity has an extensive supporting literature [8], [9], [10], [11]. While other important research focuses on the theoretical, mathematical, configurational aspects of spatial complexity, this paper focuses on visualisation of spatial complexity. While theoretical concepts of 'visuality' and 'scopic regime', relating to the ways in which both what is seen and how it is seen are socially constructed, it is sometimes suggested that the 'privileging' of the visual empowers the researcher, thus reducing objectivity. It is argued that understandings of visualisation require knowledge about provenance as well as the social work that the image does' [12]. In the field of visual research, a critical visual methodology approach [13] is proposed in the analysis of visual culture, including close attention to the actual visual artifact, thinking about the social conditions and effects of visual objects, and consideration of the researchers approach to viewing images. Visual research methods for design tend to emphasise the visual features of the built environment [14] and include evaluative responses for urban design [15], whereby general principles in aesthetics related to urban environments are sought. 
Stanczak [16] emphasises the need for reflexivity in considering visual research methods ${ }^{1}$, and this paper adopts an abductive reasoning logic approach to visualisation of spatial complexity, making reasonable and logical claims, without being definitive [17].

Data-informed urbanism [3] is seen as a key emergent phenomenon globally, as cities are increasingly the generators of big data. This form of urbanism is being complemented and replaced in some instances by data-driven, networked urbanism as 'cities are becoming ever more instrumented and networked, their systems interlinked and integrated, and vast troves of big urban data are being generated and used to manage and control urban life in real-time' [3]. In this context, developments such as 'city-dashboards' (live feeds of real-time data communicated to citizens) are improving on simply providing raw data by producing visualisations that aid interpretation and analysis, especially for non-expert users, 'allowing citizens to monitor the city for themselves' [17]. Although visualisation is not new ${ }^{2}$, visual representation of data has emerged as a significant factor in communicating complex data sets including evaluation related to urban environments. The intersection of researcher and tactics or tools is subject of debate in artistic research ${ }^{3}$ and in architecture and design [18], where tools are sometimes associated with a 'practice' rather than a 'research' aim. In defining the term 'tools' in relation to urban design evaluation processes, Gil describes the term 'tool' as 'used in a broad sense, encompassing a range of design and decision-support instruments' [19], the meaning adopted in this paper. In the broader research on spatial complexity by the author (of which this paper forms a part) ${ }^{4}$ two distinct types of 'tool' are employed: evaluation tools and visualisation tools. Only the visualisation tools are concentrated on in this paper.

\footnotetext{
${ }^{1}$ Stanczak (2007), in discussing visual research methods for social sciences, describes an epistemology of visual research methods, as follows: 'just as subjectivity and realism interact in the space between the image and the viewer, the same happens between the producer of the image and the subject or content. We may select the time and space that we want to capture, but the mechanical operation of the camera will document all that is before it in that moment. In other words, the camera is susceptible to the selectivity of the operator, but it is not selective once the shutter is opened (Collier \& Collier, 1986)' [16].

${ }^{2} \mathrm{Dr}$ Snow's linking of the spread of cholera to water supply in London in 1854 is an early example of data visualisation [20].

${ }^{3}$ Lesage (2009) describes the concept of artistic research as follows: 'The notion of artistic research implies that artistic practice can be described in a way more or less analogous to scientific research. An artistic project, then, begins with the formulation, in a certain context, of an artistic problem, which necessitates an investigation, both artistic and topical, into a certain problematic, which may or may not lead to an artwork, intervention, performance or statement, with which the artist positions himself/herself with regard to the initial artistic problem and its context' [21]. The distinction of the artistic research approach as defined here from the urban design research approach of this study is discussed in Section 4.5.2.

${ }^{4}$ See (Mee, 2017) PhD titled: 'Exploring, Evaluating and Visualising Spatial Complexity of Urban Sites'. Abstract available at https://arrow.dit.ie/appadoc/79/
} 


\subsection{Artistic practice and urban design visualisation}

This section briefly addresses art practice and urban design visualisation. In relation to evaluation results of urban design analysis, numerous researchers have recently linked urban design and artistic practice [22], [23] with a proposal that urban design could become refocused if conceived of as an integrative art of place [23]. In information design, Tufte sees the world as 'complex, dynamic, multidimensional; the paper is static, flat. How are we to represent the rich visual world of experience and measurement on mere flatland ?' [24]. In his book, 'Beautiful Evidence', Tufte emphasises the importance of including multiple types of evidence in research: 'Evidence that bears on questions of any complexity typically involves multiple forms of discourse. Evidence is evidence, whether words, numbers, images, diagrams, still or moving' [25]. Onwuegbuzie et al's presentation of a broad taxonomy of visual representation of mixed methods research includes connections made to mixed methods research outputs generally, suggesting that graphical methods have particular strengths in this regard [26]. The recent urban design research methods literature is silent on the topic of creative or artistic research methods [27]. As regards the relevance of the art of urban design, (or of 'art' to the practice of urban design) it was demonstrated in the wider study by the author, especially in the exploratory stages, that the interpretative, artistic interpretation of the infographics employed enable 'abductive' knowledge to emerge, and that this knowledge contributes to later, more hard-scientific, evaluation results. Hence, it can be concluded that the interpretative, creative aspects of urban analysis do have a place in urban evaluation and therefore do contribute to urban design practice.

\subsection{Visualisation approach and data transformation tasks}

Having discussed the links between spatial complexity, representation and visualisation theory, and then focused more directly on artistic practice and urban design visualisation, this section moves to describing the visualisation approach of this research and paper and some of the data transformation tasks undertaken. Prior evaluation of three case study urban sites in Dublin, Ireland, was undertaken in the wider study, and the approach to visualisation of results is the focus of this paper. Three sites were evaluated as having distinct and contrasting conditions of evaluated spatial complexity: low (Carmanhall), medium (Ballymun) and high (Liberties). In case study research it is a primary strategy that data sources, data types or researchers are triangulated appropriately, in order that it can be established that phenomena have been explored and viewed from multiple perspectives. Clear and accessible visual representation of this multi-scalar and multi-criteria analysis is important in this regard. Correlation and comparison of case study data enhances overall data quality based on the principles of idea convergence and the confirmation of findings [28]. The data gathered converges to illuminate the cases as well as the conditions studied in a new way. The fact that indices of spatial complexity vary related to time, scale and geography, and that these necessarily vary in each demonstrative case 
study unit, means that linkages between cases and indices become a rich source of descriptive account of phenomena.

In information design, a distinction is made between data visualization and infographics, whereby the former is associated with algorithmic generation and the latter with manually generated images. Data visualization, in categorization terms, is considered to have two types, exploration and explanation (lliinsky et al, 2011:7), and each suggests different approaches and tools. So while exploratory data visualizations are associated with high levels of granularity, where large amounts of data are in play, at the data analysis phase of a project, the narrative emerging from the data is still to be set. Explanatory data visualizations, in contrast, are seen as connected more to facts which are already known to the designer/researcher, and to reporting more concrete results, and as part of the presentation phase of a project.

However, Iliinsky [29] also proposes a third category, which is useful to this study, the hybrid 'exploratory explanation data visualization', seen as 'a curated dataset' [29], which is presented in a way that allows the reader to interact with the dataset in some way. Information, persuasion and visual art are also considered relevant and important in understanding concepts of data visualization [29], and it is in this respect that the connection between data visualizations and urban design as art becomes important to this study. Iliinsky [29] suggests that there are three main categories of explanatory visualizations based on the relationships between the three necessary players: the designer, the reader, and the data, considered as three essential supports to effective explanatory (or hybrid) data visualization. However, the dominant relation between two of these elements will determine the type of data visualization needed. Informative, persuasive and 'visual art' data visualizations are considered the three types to consider in deciding on data visualization type or categorisation. So while informative visualizations distill information into consumable form (eg. for a newspaper), persuasive type visualizations seek to change a readers mind about something, from a specific point of view. The third category, visual art, is considered to serve primarily the relationship between the designer and the data [29]. Visual art is considered to be 'unidirectional' in form, that is, the reader may not be able to decode the visual presentation to understand the underlying information [29]. So while 'both informative and persuasive visualizations are meant to be easily decodable-bidirectional in their encoding-visual art merely translates the data into a visual form' [29]. From the review of the data visualization literature, it is concluded that the majority of the visual representations in this study are in the hybrid 'exploratory explanation data visualization' category. Exploratory infographics are especially employed in the exploratory 'whole-city' explorations of spatial complexity. However, exploratory data visualizations are also derived and employed. These are especially used in visualising the case study evaluations, to further informative and persuasive aims of the overall study. As regards data transformation tasks, three key tools for visualisation of the evaluation of spatial complexity are employed: Tables, Plots and Boxes. Each is employed both in linear, consecutive fashion (Table prepared first, then Plot, etc) but also all three can be used simultaneously and iteratively (for example in urban designing) in a complexity frame. 


\subsection{Evaluation Table}

An Evaluation Table is proposed as the first key tool of visualisation of the evaluation of spatial complexity. This tool has two data dimensions (across top of Table and vertically along one side), is updated manually and results in a shallow, infographic type output. In terms of data transformation [30], quantification of qualitative data is shown in the Spatial Complexity Evaluation Table, where urban morphological complexity analysis, though including 'metrics' also has a more qualitative, text driven aspect, leading to evaluation. In this study, qualification of quantitative data involves interpretation of mathematical measures of spatial complexity (for example 'integration'), broadening descriptions into high, medium or 'low'. In order to visualise that equal weightings applying to these criteria are interrelated, and in order to allow comparisons across cases, colour weightings are applied to the Table. A matrix is recommended in data analysis for comparison of quantitative and qualitative data. In this research, the primary matrix-type evaluation tool takes the form of the Spatial Complexity Evaluation Table, and (at a lower level) a separate matrix of compositional complexity metrics, represented as a Table of Compositional Criteria. The separate matrices allow that data can be compared within, between and across urban sites, but also with other data from previous studies of the constituent criteria of spatial complexity. In this way, individual characteristics of the sites (like for example, evaluated density) can be compared, or the spectrum of densities evaluated across three urban sites in Dublin could be compared directly with international examples, in order that proposed design densities are shown to be appropriate. This is an example of the first of four particular benefits to urban design practice of employing appropriate visualisation of spatial complexity: to stimulate urban design process.

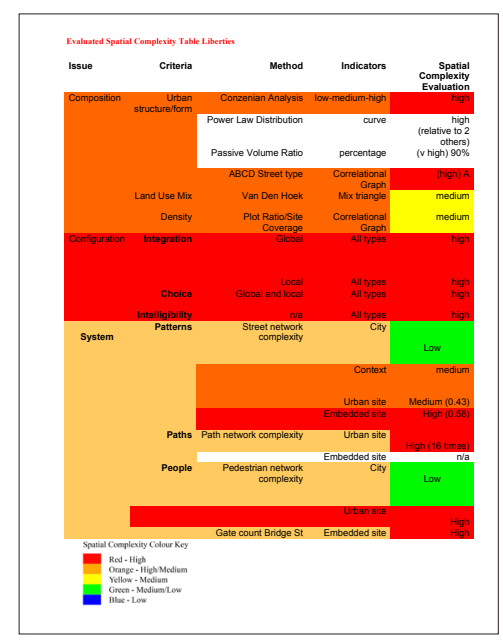

Fig. 1. Coloured Spatial Complexity Evaluation Table 


\subsection{Spider Plot}

Spider plot is proposed as the second key tool of visualisation of the evaluation of spatial complexity. This tool has nine data dimensions (around a centre of the graphical web, nine evaluated criteria of spatial complexity are recorded graphically, from low, at the centre, to high, at the edge). Although this graphic is updated manually it results in a relatively medium rich 'data depth', but still exists as an infographic type output. As regards visualization tools in the decision-making process related to evaluation, in particular for complex issues, so-called 'spider analysis' is recommended for comparative and scenario studies [31]. Defined as 'an analytical tool, which can be used to visualize the relative strengths and weaknesses of the selected case studies or different scenarios for various chosen factors' [32]. It functions, not as a mathematical tool, but as a visual analysis instrument. The 'spider' refers to the appearance of a spider's web, and the scores of each factor are plotted on an axis which has lowest scores at the centre, working outwards towards higher values. The resulting image is called a spider plot. According to Baycan-Levent, the scores may be qualitative (ie. ordinal rankings) or quantitative (eg. standardized on a 10 point scale). In recent urban design evaluation, Serra, Gil, \& Pinho [33] have used this model to illustrate and apply a 'taxonomic nomenclature' evolving street patterns, and Mehta has used this format to visualize indices of evaluation of public space. Mehta argues that the value of the index developed is 'not in absolute values or scores but by the graphic representation of the spaces' [34]. A spider plot format can visually summarise both the quantitative and qualitative aspects of the urban site evaluations, and in simple form can be based on either lines alone, or lines and colour. Quick summary impressions of relative levels of evaluated spatial complexity can be achieved with spider plots, and visual comparisons within, between and across urban sites are improved by spider plot preparation. This is an example of the second of four particular benefits to urban design practice of employing appropriate visualisation of spatial complexity: to bring visual clarity to quantitative assessment.

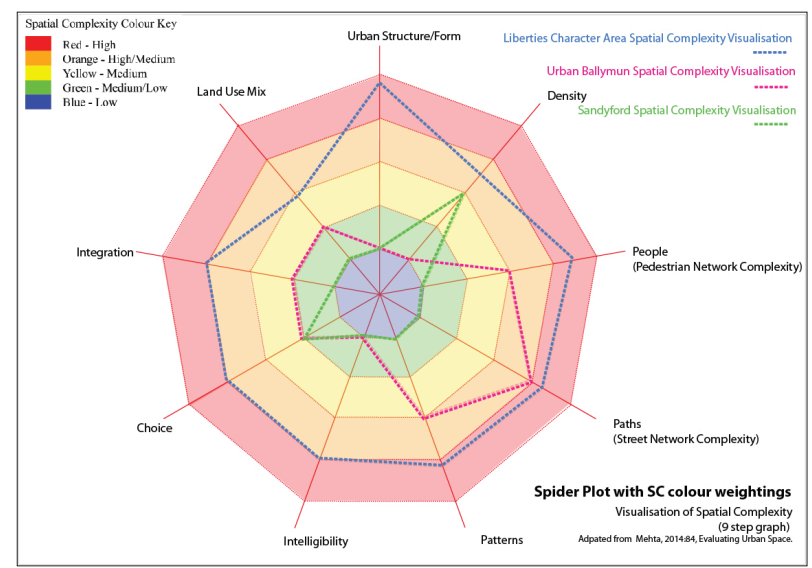

Fig. 2. Coloured Spatial Complexity Evaluation Table 


\subsection{Databox}

A Databox is proposed as the third key tool of visualisation of the evaluation of spatial complexity. This Spatial Complexity Databox tool has thirty-six data dimensions (three spatial dimensions, $X, Y$ and $Z$, as axes of a display cube, time as a fourth 'dimension', and nine evaluated criteria of spatial complexity, recorded graphically in 'heat-map' terms, on volumetric digital models of urban blocks and structures). The incorporation of a temporal dimension adds a dynamic aspect to the tool. This graphic could be updated automatically and algorithmically. It results in a rich 'data depth', and therefore can be categorised as an explanatory data visualisation type output. The concept of a 'spatial data cube' is defined as a unit of organisation of spatial data which facilitates data mining and 'organization of data into multidimensional structures and hierarchies' [35]. Two important components of the Spatial Complexity Databox, the 'voxel' and a 'spatiotemporal database', are now described. Hong's conceptualization of the 'voxel' or 'volumetric pixel' was developed in order to facilitate a theory of 'interdependant urbanism', which involves, in the authors terms, 'simulating interdependent complexity, beyond prescriptive zoning' [36]. Hong suggests that this innovation can improve on land use and development zoning in cities. Hong also argues that computation can play a major role in urban design, 'by leveraging performance based zoning standards instead of prescriptive rules'. His innovation is in representing performance of the urban environment through 'simultaneous evaluation' of variables ('daylighting, building cores, proximity to parks, programming and other factors') in order to give immediate feedback to designers, planners and stakeholders about the existing urban environment as well as potential urban design scenarios. He employs Rhinoceros and its Grasshopper plug-in software in conjunction with the programming language Python. After describing a number of novel tools which pertain to 'the generation of maximum envelopes' of urban form (for example seek optimal design forms related to sunlight and sky exposure) Hong goes on to discuss the idea of the 'voxel' (short for volumetric pixel) as a way to subdivide this overall mass and imbue it with qualitative data in the form of both inputs and outputs ${ }^{5}$. In another innovative approach to visualization of spatial data, Hahn et al define a 'spatiotemporal database' as a 'spatial database that stores spatial objects that change with time' [35]. These trends in urban data visualization are reflected in other enquiries, including investigations from architecture like 'information urbanism', [37] and 'parametric urbanism' [38], and geographical research into 'data-driven, networked urbanism' [15] though the latter is still primarily represented in two dimensional plan. These two concepts, of the 'voxel' and a 'spatio-

\footnotetext{
${ }^{5}$ Hong describes the advantages of the voxel as follows; 'Designers have the freedom to assign any number of parameters to the voxels, limited only by computation power. For our test case we included such factors as minimum daylight factor, views, circulation, and proximity to open space. From these inputs, qualitative outputs, or 'readings', of data are produced. The voxels thereby become an interconnected mesh, as data output from one voxel can be fed into the input of another, allowing interdependencies to ripple through the entire model.' [36].
} 
temporal database' are incorporated within the idea of the Spatial Complexity Databox proposed in this paper. The Databox is in digital form, and can be manipulated by any user of the interface, with no technical or professional training required. This is an example of the third of four particular benefits to urban design practice of employing appropriate visualisation of spatial complexity: to allow qualitative interpretation by a wide community around evaluation.

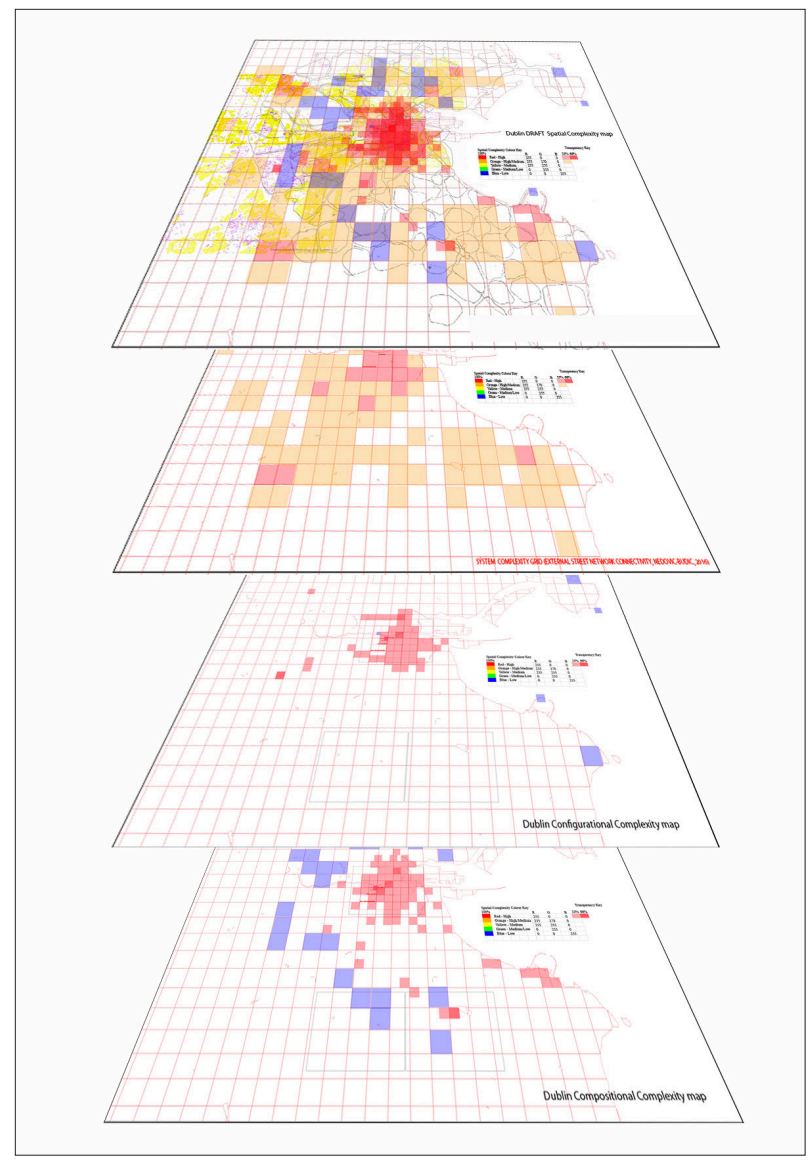

Fig. 3. Spatial Complexity Databox

\section{Discussion}

In summary, three key tools are proposed for visualisation of the evaluation of spatial complexity: Tables, Plots and Boxes. As described, all three can be used simultaneously and iteratively to represent evaluation, but also have other applications (for example in urban designing) within a complexity frame. Seen together, these visualisations graphically establish a unique 'signature of spatial complexity' for each urban 
site. The visualisation involves communicating quantitative analysis and also qualitative data, judgement in graphically representing and joining both, and combining these in a visually clear form. This is an example of the last of four particular benefits to urban design practice of employing appropriate visualisation of spatial complexity: to allow formal measurement and informal interpretation of results to be combined.

\section{Conclusions}

Spatial complexity (the spatial component of urban complexity) has previously been visually represented in hard-scientific domains through pattern recognition in pixels of aerial views at large scales [4] classification of rural landscapes through macro scale mapping [5], and recognition of fractal or geometrical patterns across populations, remote sensed imagery and street network representations [6].However, these approaches tend towards a single scale of analysis, single disciplinary applications, and are fixed or 'static' snapshots of a moment in time. This paper (and the wider study) confirms that dynamic visualisation of spatial complexity incorporating some key tools can improve on more 'hard-scientific' (or 'science of cities') evaluation of urban sites, related to description of evaluation. In creatively ${ }^{6}$ visualising spatial complexity, multi-scalar, relational and temporal aspects of the urban built environment can be captured. It is shown that artistic visualisation of spatial complexity contributes to improved urban design practice and more detailed understanding of existing urban site conditions. Furthermore, it is demonstrated that visualisation of evaluation of urban sites matters to urban design. Four particular benefits to urban design practice of employing appropriate visualisation of spatial complexity are suggested. Firstly, to stimulate urban design process: secondly, to bring visual clarity to quantitative assessment: thirdly, to allow qualitative interpretation by a wide community around evaluation, and lastly, to allow formal measurement and informal interpretation of results to be combined, which in turn involves a wider group of stakeholders around evaluation.

6 In defining what constitutes 'creative' in this context, Caliskan (2012) [39], distinguishes between artistic or creative invention (essentially independant of any functional or pragmatic incentives) and Gero's definition of 'design' ('goal-oriented, constrained decision-making, exploration and learning activity that operates within a context that depends on the designer's perception') (Gero, 1990 ) [40][39]. 


\section{References}

1. Barredo JI, Kasanko M, McCormick N, et al. (2003) Modelling dynamic spatial processes: simulation of urban future scenarios through cellular automata. Landscape and Urban Planning 64: 145-160.

2. Rydin Y, Bleahu A, Davies M, et al. (2012) Shaping cities for health: complexity and the planning of urban environments in the 21st century. Lancet 379: 2079-2108.

3. Kitchin R. (2015) Data-driven, networked urbanism. Data and the City Workshop, Maynooth, Co. Kildare, Ireland.

4. Haghani T. (2010) Fractal Morphology \& Urban Complexity. Phd Thesis, School of Architecture, BIAD, Birmingham City University (BCU), UK.

5. Laterra P, Orúe ME and Booman GC. (2012) Spatial complexity and ecosystem services in rural landscapes. Agriculture, Ecosystems \& Environment 154: 56-67.

6. Batty M. (2011) Building a Science of Cities. Cities: 1-8.

7. Bhat R. (2014) Understanding complexity through pattern languages in biological and man-made architectures. 2014 8: 10.

8. Lynch K. (1984) Reconsidering the image of the city: Springer.

9. Heath T, Smith S and Lim B. (2000) The complexity of tall building façades. Journal of Architectural \& Planning Research 17: 206-220.

10. Tucker C, Ostwald M, Chalup S, et al. (2005) A Method for the Visual Analysis of the Streetscape. University of Newcastle.

11. Ostwald MJ, Vaughan J and Tucker C. (2015) Characteristic visual complexity: Fractal dimensions in the architecture of Frank Lloyd Wright and Le Corbusier. Architecture and Mathematics from Antiquity to the Future. Springer, 339-354.

12. Fyfe $G$ and Law J. (1988) Picturing power: visual depiction and social relations: Routledge.

13. Rose G. (2007) Visual Methodologies An Introduction to the Interpretation of Visual Materials, London: Sage Publications.

14. Sanoff H. (2016) Visual Research Methods in Design (Routledge Revivals): Routledge.

15. Nasar JL. (1994) Urban design aesthetics the evaluative qualities of building exteriors. Environment and Behavior 26: 377-401.

16. Stanczak GC. (2007) Visual Research Methods: Image, Society, and Representation: SAGE Publications.

17. Kitchin R. (2014) Big Data, new epistemologies and paradigm shifts. Big Data \& Society 1.

18. Nilsson F and Dunin-Woyseth H. (2014) Design Education, Practice, and Research: On Building a Field of Inquiry. Studies in Material Thinking 11.

19. Gil J, Duarte, José. (2013) Tools for evaluating the sustainability of urban design: a review. Proceedings of the ICE - Urban Design and Planning 166: 311-325.

20. Minty E, Maccallum P, Fisher J, et al. (1995) Scientific Visualisation: A Practical Introduction.

21. Lesage D. (2009) Who's Afraid of Artistic Research? On measuring artistic research output. A Portrait of the Artist as a Researcher. Antwerp: The Academy and the Bologna Process/MuHKA: 84-93.

22. Boyko CT, Cooper R, Davey CL, et al. (2010) Informing an urban design process by way of a practical example.

23. Marshall S. (2015) Refocusing urban design as an integrative art of place. Proceedings of the Institution of Civil Engineers Urban Design and Planning 168: 8-18.

24. Tufte ER. (1990) Envisioning Information, Cheshire, Connecticut, USA: Graphics Press.

25. Tufte ER. (2006) Beautiful evidence. New York. 
26. Onwuegbuzie AJ and Leech NL. (2007) Validity and qualitative research: An oxymoron? Quality \& Quantity 41: 233-249.

27. Carmona ME. (2014) Explorations in Urban Design An Urban Design Research Primer, United Kingdom: Ashgate Publishing Ltd.

28. Knafl KA and Breitmayer BJ. (1989) Triangulation in qualitative research: Issues of conceptual clarity and purpose. Qualitative nursing research: A contemporary dialogue: 193-203.

29. Iliinsky N and Steele J. (2011) Designing data visualizations: Representing informational Relationships: O'Reilly Media, Inc..

30. Cresswell JW. (2009) Research Design Quantitative, Qualitative, and Mixed Methods Approaches, London: Sage.

31. Baycan-Levent T. (2005) Urban spiders: a comparative framework for evaluation and scenario analysis. Sustainable Urban Development: The environmental assessment methods. 236.

32. Rienstra SA and Nijkamp P. (1998) The role of electric cars in Amsterdam's transport system in the year 2015; a scenario approach. Transportation Research Part D: Transport and Environment 3: 29-40.

33. Serra M, Gil, Jorge, Pinho, Paulo. (2013) Unsupervised classification of evolving metropolitan street patterns. In: Y O Kim HTPaKWS (ed) Ninth International Space Syntax Symposi$u m$. Sejong University, Seoul.

34. Mehta V. (2013) Evaluating Public Space. Journal of Urban Design 19: 53-88.

35. Han J, Kamber M and Pei J. (2011) Data mining: concepts and techniques: Elsevier.

36. Hong J. (2012) Interdependent Urbanism. In: Schröpfer T (ed) Ecological urban architecture: qualitative approaches to sustainability. Walter de Gruyter, $140-150$.

37. Tang $M$ and Anderson J. (2014) Information Urbanism: Parametric urbanism in junction with GIS data processing \& fabrication. ARCC Conference Repository.

38. Schumacher, P., 2009. Parametricism: A new global style for architecture and urban design. Architectural Design, 79(4), pp.14-23.

39. Çaliskan O. (2012) Design thinking in urbanism: Learning from the designers. Urban Design International 17: 272-296.

40. Gero JS. (1990) Design prototypes: a knowledge representation schema for design. Al magazine 11: 26. 